\title{
Endostar-loaded PEG-PLGA nanoparticles: in vitro and in vivo evaluation
}

\author{
This article was published in the following Dove Press journal: \\ International Journal of Nanomedicine \\ 23 November 2010 \\ Number of times this article has been viewed
}

\author{
Sanyuan $\mathrm{Hu}^{\prime}$ \\ Yangde Zhang ${ }^{2}$ \\ 'Xiangya School of Medicine and \\ ${ }^{2}$ National Hepatobiliary and Enteric \\ Surgery Research Center, Ministry \\ of Health, Central South University, \\ Changsha, Hunan Province, People's \\ Republic of China
}

\begin{abstract}
Endostar, a novel recombinant human endostatin, which was approved by the Chinese State Food and Drug Administration in 2005, has a broad spectrum of activity against solid tumors. In this study, we aimed to determine whether the anticancer effect of Endostar is increased by using a nanocarrier system. It is expected that the prolonged circulation of endostar will improve its anticancer activity. Endostar-loaded nanoparticles were prepared to improve controlled release of the drug in mice and rabbits, as well as its anticancer effects in mice with colon cancer. A protein release system could be exploited to act as a drug carrier. Nanoparticles were formulated from poly (ethylene glycol) modified poly (DL-lactide-co-glycolide) (PEGPLGA) by a double emulsion technique. Physical and release characteristics of endostar-loaded nanoparticles in vitro were evaluated by transmission electron microscopy (TEM), photon correlation spectroscopy (PCS), and micro bicinchoninic acid protein assay. The pharmacokinetic parameters of endostar nanoparticles in rabbit and mice plasma were measured by enzyme-linked immunosorbent assay. Western blot was used to detect endostatin in different tissues. To study the effects of endostar-loaded nanoparticles in vivo, nude mice in which tumor cells HT-29 were implanted, were subsequently treated with endostar or endostar-loaded PEG-PLGA nanoparticles. Using TEM and PCS, endostar-loaded PEG-PLGA nanoparticles were found to have a spherical core-shell structure with a diameter of $169.56 \pm 35.03 \mathrm{~nm}$. Drug-loading capacity was $8.22 \% \pm 2.35 \%$ and drug encapsulation was $80.17 \% \pm 7.83 \%$. Compared with endostar, endostar-loaded PEG-PLGA nanoparticles had a longer elimination half-life and lower peak concentration, caused slower growth of tumor cell xenografts, and prolonged tumor doubling times. The nanoparticles changed the pharmacokinetic characteristics of endostar in mice and rabbits, thereby reinforcing anticancer activity. In conclusion, PEG-PLGA nanoparticles are a feasible carrier for endostar. Endostar-loaded PEG-PLGA nanoparticles seem to have a better anticancer effect than conventional endostar. We believe that PEG-PLGA nanoparticles are an effective carrier for protein medicines.
\end{abstract}

Keywords: medical physics, biologic physics, nanoparticles

\section{Background}

Cancer affects millions of men and women in all age groups. Colorectal cancer is one of the most common internal malignancies. However, many conventional chemotherapies are ineffective in colorectal cancer, and in many other cancers, because of short halflives and inability to reach the tumor site in effective concentrations. ${ }^{1}$ The emergence of nanotechnology has had a profound effect on chemotherapy for cancer. To date, many anticancer drugs have been incorporated into polymeric micelles, surface-modified particles, liposomes, or nanoparticles for delivery to the tumor. ${ }^{2,3}$ There have been several problems with these approaches, including limited biodistribution, toxic side 
effects, and rapid clearance by the reticuloendothelial system. However, nanoparticles are different from other drug carriers, with many potential chemotherapeutic advantages, including convenient injection, deposition in target tissues, an enhanced targeting effect in primary or metastatic tumors, and reduction of toxicity to normal tissues. ${ }^{4-6}$ In addition, poly(ethylene glycol) modified poly (DL-lactideco-glycolide) (PEG-PLGA) nanoparticles can incorporate water-soluble anticancer drugs and reduce drug interaction within the reticuloendothelial system. Moreover, poly(DLlactide-co-glycolide) (PLGA) and poly(ethylene glycol) (PEG), which are hydrophilic-hydrophobic diblock copolymers, are biodegradable, nontoxic, ${ }^{7}$ nonimmunogenic, and often act as drug incorporation sites.

Endostatin, a $20 \mathrm{kDa}$ internal fragment of the carboxyterminus of collagen XVIII, has been demonstrated to inhibit the growth of a variety of human tumors by inhibiting neovascularization. ${ }^{8,9}$ However, most available endostatins are either unstable or expensive, which limits their clinical application. Endostar, a novel recombinant human endostatin, was expressed and purified in Escherichia coli. It was approved by the Chinese State Food and Drug Administration for the treatment of nonsmall cell lung cancer in 2005 and has a broad spectrum of activity against solid tumors. Endostar had been shown to inhibit endothelial cell proliferation, migration, and vessel formation. ${ }^{9}$ Moreover, it is more stable than conventional endostatin, because it has an additional nine-amino acid sequence at the $\mathrm{N}$ terminus of the protein. ${ }^{10,11}$

In this study, we hypothesized that PEG-PLGA nanocarrier systems could increase the circulation half-life of endostar by exploiting the enhanced permeation retention phenomenon effectively, and thus increase the effect of the drug.

\section{Materials and methods Materials}

Endostar $(5 \mathrm{mg} / \mathrm{mL})$ was provided by Shandong Simcere Medgenn Bio-Pharmaceutical Co Ltd (Nanjing, China). PEG-PLGA was purchased from the Shandong Institute of Medical Instruments (Shandong, China). The molar ratio of D, L-lactic to glycolic acid of PLGA (molecular weight $45 \mathrm{kDa}$ ) was 50:50. Every $10 \mathrm{~g}$ of PEG-PLGA contained $1 \mathrm{~g}$ PEG (molecular weight $2 \mathrm{kDa}$ ). Polyvinyl alcohol (molecular weight 13-23 kDa) was obtained from Sigma-Aldrich (St Louis, MO). The primary antibodies for CD-31, endostatin, vascular endothelial growth factor, and $\beta$-actin were purchased from Santa Cruz Biotechnology (Santa Cruz, CA).
The S-P detection kit was purchased from Fuzhou Maixin Co (Fujian, China). All other chemicals were analytic grade. Double-distilled water was used throughout the study. The apparatus, including a low-temperature ultracentrifuge (Hitachi, Japan), transmission electron microscopy (TEM, Philips, The Netherlands), photon correlation spectroscopy (PCS, Malvern, UK), ultraviolet spectrophotometer (Spectrum China Ltd, Shanghai, China), and an enzyme-linked immunosorbent assay (ELISA) reader (BioTek, Winooski, VT) were used. New Zealand rabbits (2-3 kg) and BALB/c nude mice (4-6 weeks of age, 10-25 g) were purchased from the Animal Center of Shandong University, Shangdong, China, and Hunan Slac Laboratory Animal Co Ltd, Hunan, China, respectively. All work performed with the animals was in accordance with and approved by the ethics committee of Shandong University.

\section{Synthesis of PEG-PLGA nanoparticles}

PEG-PLGA nanoparticles were prepared by a double emulsion (mixing solvent) method as described in previous studies. ${ }^{12,13}$ Firstly, PEG-PLGA was dissolved in dichloromethane. The first emulsion $(\mathrm{o} / \mathrm{w})$ was formed between a dichloromethane solution of PEG-PLGA $(1 \mathrm{~mL})$ and endostar solution $(0.05 \mathrm{~mL})$ by shearing (2800 rpm for 60 seconds). Then a $2 \mathrm{~mL}$ aqueous solution containing $0.1 \%$ polyvinyl alcohol was added into this primary w/o emulsion to obtain the double emulsion (w/o/w) by high-speed shearing (25000 rpm for 60 seconds). The solvent was evaporated in aqueous solution $10 \mathrm{~mL}$ containing $0.1 \%$ polyvinyl alcohol $(\mathrm{w} / \mathrm{v})$ either by gentle magnetic stirring at room temperature or using a vacuum rotating evaporator. Nanoparticles were recovered by centrifugation (40000 rpm for 40 minutes) and washed three times in phosphate-buffered saline.

\section{Entrapment efficiency}

After dissolving the lyophilized nanospheres in $0.05 \mathrm{~N} \mathrm{NaOH}$ and $1 \%$ sodium dodecyl sulfate, the endostar content of the nanoparticles was estimated using the micro bicinchoninic acid protein assay. ${ }^{14}$ No interference with the PEG-PLGA or stabilizers was observed. The assay was validated using purified recombinant human endostatin with a detection limit of $0.5-20 \mu \mathrm{g} / \mathrm{mL}$. The concentration of endostar in the supernatant $(\mathrm{C})$ was calculated according to the standard curve equation. The drug-loading capacity and entrapment efficiency were expressed as follows:

$$
\begin{gathered}
\text { Endostar loading capacity }(\%) \\
=\mathrm{M}_{\text {endostar }} / \mathrm{M}_{\text {endostar-loaded PEG-PLGA nanoparticles }} \times 100 \\
\text { Endostar encapsulation }(\%)=\mathrm{M}_{\text {endostar }} / \mathrm{M}_{\text {endostar devoted }} \times 100
\end{gathered}
$$


$M_{\text {endostar }}$ was the drug amount in the nanoparticles $\left[\mathrm{M}_{\text {endostar }}=\mathrm{C} \times \mathrm{V}, \mathrm{C}\right.$ : concentration in the supernatant, $\mathrm{V}$ : volume]; $\mathrm{M}_{\text {endostar-loaded }}$ PEG-PLGA nanoparticles was the amount of PEG-PLGA nanoparticles containing endostar; and $\mathrm{M}_{\text {endostar devoted }}$ was the initial amount of Endostar.

\section{Particle size analysis}

Morphologic examination of the nanoparticles was performed using TEM. Particle size distribution, ie, mean diameter and polydispersity index (PDI) was determined by PCS. The PDI was calculated as follows:

$\mathrm{PDI}=\mathrm{M}_{\mathrm{w}} / \mathrm{M}_{\mathrm{n}} \cdot\left(\mathrm{M}_{\mathrm{w}}\right.$ : the weight average molecular weight; $M_{n}$ : the number average molecular weight)

The presence of residual polyvinyl alcohol on the surface of the nanoparticles was determined by direct and indirect methods..$^{15}$ The nanoparticles were digested in $0.05 \mathrm{~N} \mathrm{NaOH}$ and $1 \%$ sodium dodecyl sulfate. The solution obtained was then neutralized using $\mathrm{HCl}$ diluted with phosphate-buffered saline ( $\mathrm{pH}$ 7.4), and analyzed for polyvinyl alcohol content using the colorimetric method. Absorbance of the formation of polyvinyl alcohol-iodine complexes was determined in the presence of boric acid at $620 \mathrm{~nm}$. The residual polyvinyl alcohol was also calculated according to the difference between the total amount used and the amount present in the supernatant of the washing steps. Corrections were made for the PEG-PLGA nanoparticles, because PEG resulting from polymer degradation would interfere with the polyvinyl alcohol dosage.

\section{In vitro release}

Endostar-loaded nanoparticles were washed three times with phosphate-buffered saline and added to a dialysis Eppendorf tube with phosphate-buffered saline $(\mathrm{pH} 7.4,0.01 \%$ sodium azide, $0.02 \%$ Tween 80 ). The Eppendorf tube was stirred at $100 \mathrm{rpm}$ and at $37^{\circ} \mathrm{C}$. At indicated time intervals, the suspension was centrifuged at $40000 \mathrm{rpm}$ for 40 minutes. ${ }^{14}$ The supernatant was removed and detected using the micro bicinchoninic acid protein assay with an ultraviolet spectrophotometer at $562 \mathrm{~nm}$, and new phosphate-buffered saline dialysis media was added to the Eppendorf tube. The endostar released into the replaced phosphate-buffered saline was calculated at different time intervals according to the standard curve, which was established by purified recombinant human endostatin. The release curve of endostar-loaded nanoparticles was then described.

\section{Pharmacokinetic study of endostar-loaded nanoparticles in vivo} Forty male BALB $/ \mathrm{c}$ mice (mean weight $0.021 \mathrm{~kg}$ ) were administered a single intravenous bolus of endostar or endostar-loaded nanoparticles via the tail vein at a dose of $90 \mathrm{mg} / \mathrm{m}^{2}(\mathrm{n}=20$ per dose group). Blood samples were collected by retro-orbital bleeding from the two groups at the indicated time intervals. Because blood samples could not be collected from the same mouse repeatedly over a short time period, several mice died after collection of blood, and New Zealand rabbits were used subsequently. Ten New Zealand rabbits were randomized into two groups. After 12 hours of fasting, a bolus of the sample equivalent to $90 \mathrm{mg} / \mathrm{m}^{2}$ endostar or endostar-loaded nanoparticles, was administered intravenously to each rabbit. Each rabbit was given an equal amount of endostar intravenously. Blood samples were collected from the aural vein at the indicated time intervals. After centrifugation, the plasma supernatant was detected by ELISA. The assay was validated using purified recombinant human endostatin with a detection limit of $2-500 \mathrm{ng} / \mathrm{mL}$. The concentrations of endostar were calculated according to the standard curve, established using a standard endostar solution. The pharmacokinetic parameters for Endostar distribution were calculated by using the DAS 2.0 program. ${ }^{16}$

\section{Amount of endostatin in different tissues in vivo}

In order to determine the clearance efficacy in the body, the amounts of endostar in different tissues were analyzed by Western blot assay using a polyclonal antiendostatin antibody. ${ }^{16}$ Male BALB/c mice were used in this step. Each mouse was administered an intravenous dose once and then sacrificed at predetermined times (10 minutes or three hours). Tissue samples of liver, spleen, and lung were collected, weighed, and homogenated. The supernatant protein were then concentrated and subjected to sodium dodecyl sulfatepolyacrylamide gel electrophoresis followed by Western blot assay with a polyclonal antihuman endostatin antibody.

\section{Tumor inhibition effect of endostar-loaded nanoparticles in vivo}

The therapeutic efficacy and biocompatibility of endostarloaded nanoparticles was evaluated in vivo by using a cancer model. HT-29 colon cancer cells suspended at a density of $10^{8}$ cells $/ \mathrm{mL}$ were inoculated subcutaneously in the right flank of BALB/c nude mice. ${ }^{17}$ After xenografts reached about $5 \mathrm{~mm}$ in diameter, the nude mice were randomly assigned to four treatment groups ( $\mathrm{n}=8$ each), ie, control, endostar, endostarloaded nanoparticles, and blank PEG-PLGA nanoparticles. Then, based on clinical dosing in humans, endostar $7.5 \mathrm{mg} / \mathrm{m}^{2} /$ day was administered intravenously once a day during the first two weeks of the treatment cycle. Endostar-loaded 
nanoparticles containing the same amount of endostar were injected every seven days to investigate the effect of controlled release in the endostar-loaded nanoparticle group. In the control group, the same volume of phosphate-buffered saline or blank PEG-PLGA nanoparticles was injected. Mice were sacrificed on day 21. During the program, tumor size was measured by calipers (length and width) every three days. The tumor volume $\left(\mathrm{V}=1 / 2 \times\right.$ length $\times$ width $\left.^{2}\right)$ was calculated and the tumor growth curve was generated. ${ }^{17,18}$ The tumor doubling time during the logarithmic phase of tumor growth and inhibition rate on day 21 was calculated. The inhibition rate was calculated as follows:

$$
\begin{aligned}
\text { Inhibition rate }(\%)= & \left(1-\text { Volume }_{\text {of experiment group }} /\right. \\
& \text { Volume } \left.e_{\text {of control group }}\right) \times 100
\end{aligned}
$$

In addition, the amount of endostar and vascular endothelial growth factor in the tumors was also analyzed by Western blot assay.

\section{Immunohistochemistry}

Tumor tissue specimens were fixed in neutral formalin and embedded in paraffin after collection from the sacrificed mice. Tissue sections $5 \mu \mathrm{m}$ thick were dewaxed and incubated with $0.01 \mathrm{M}$ natrium citricum for antigen retrieval. The slides were rinsed in phosphate-buffered saline and incubated overnight at $4^{\circ} \mathrm{C}$ with diluted anti-CD31 antibody. Steps were then performed using the immunostain kit according to the manufacturer's instructions. The sections were examined at a magnification of 100 (10 objective and 10 ocular lens) under a light microscope to identify three regions with the highest microvascular density. Microvessels were counted in these areas at a magnification of $200 \times$, and the average numbers of microvessels were recorded. The average number was the microvascular density of the tumor. ${ }^{19}$

\section{Statistical analysis}

In all cases, experiments were done in triplicate and data represented as mean \pm standard deviation. The inhibitory effect on tumor growth was analyzed by one-way analysis of variance and Student's $t$-test. ${ }^{18} P<0.05$ was considered statistically significant in all cases.

\section{Results}

\section{Characteristics of endostar-loaded nanoparticles}

In this study, the standard calibration curve equation for the concentration of endostar in the supernatant (C) was assayed using the micro bicinchoninic acid protein assay:

$$
\begin{gathered}
\mathrm{OD}=0.004 \mathrm{C}+0.0556(\mathrm{n}=3, \mathrm{r}=0.9996)(\mathrm{OD}, \text { optical } \\
\text { density value of absorbance }) .
\end{gathered}
$$

The lower limit of determination was $0.5 \mu \mathrm{g} / \mathrm{mL}$. The intraday relative standard deviation and interday relative standard deviation were less than $5 \%$, justifying use of this method for measurement of endostar concentration. Drug loading capacity was $8.22 \% \pm 2.35 \%$ and drug encapsulation of the endostar-loaded nanoparticles was $80.17 \% \pm 7.83 \%$. The morphology of endostar-loaded nanoparticles was found to be a spherical core-shell structure with a relatively smooth surface (Figure 1). It was approximately $169.56 \pm 35.03 \mathrm{~nm}$ in diameter (Figure 2). The PDI was $0.47 \pm 0.18$ by photon correlation spectroscopy. Residual polyvinyl alcohol on the surface was not detected by the two different methods.

\section{Release of endostar-loaded nanoparticles in phosphate-buffered saline}

Endostar release from the endostar-loaded nanoparticles in phosphate-buffered saline ( $\mathrm{pH}$ 7.04) is shown as a standard curve equation for the endostar solution in Figure 3. The endostar release profile was biphasic, with an initial abrupt

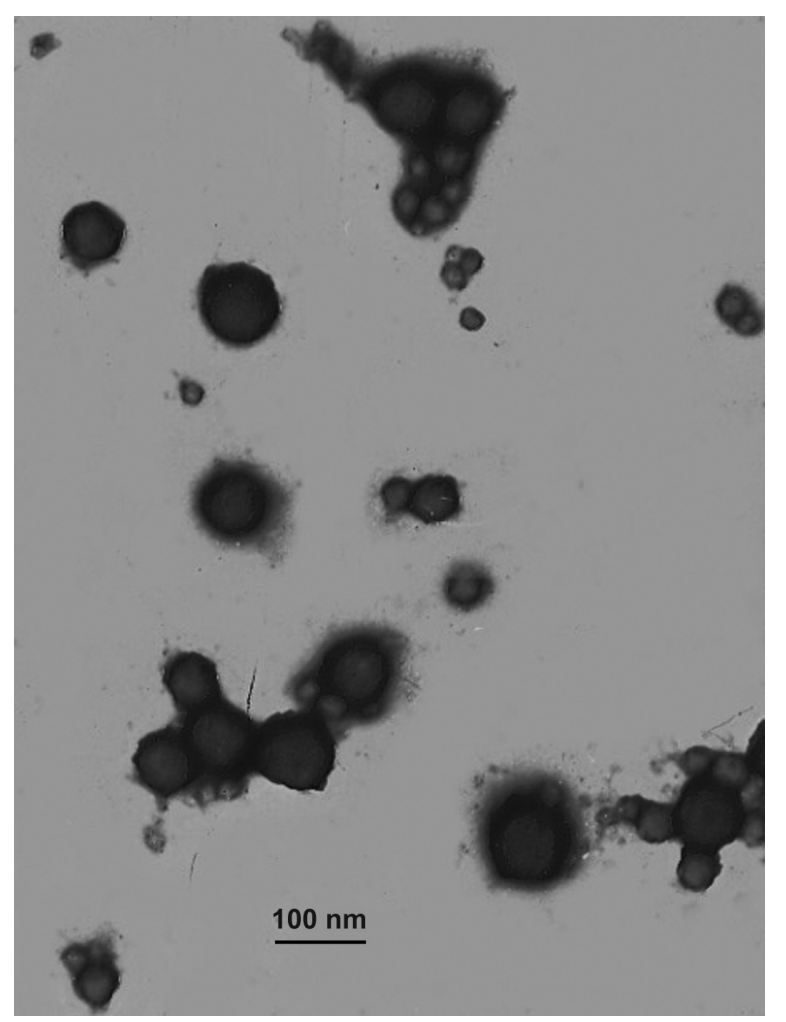

Figure I The core-shell structure of endostar-loaded PEG-PLGA nanoparticles. Transmission electron microscopy showed that nanoparticles were round particles with relative smooth edges.

Abbreviation: PEG-PGLA, poly(ethylene glycol) modified poly(DL-lactide-coglycolide). 


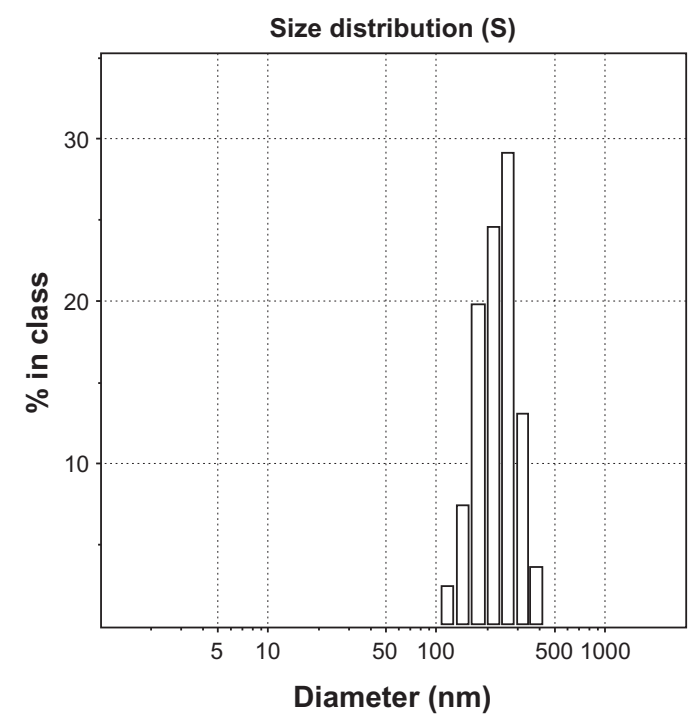

Figure 2 The distribution of nanoparticle diameter.

release and a subsequent sustained release. Abrupt release occurred at two hours after injection, and 5.0\% $\pm 1.09 \%$ of the loaded endostar was released after the first 24 hours. Almost $90 \%$ of the loaded endostar was still enveloped in the nanoparticles after 96 hours. The amount of cumulated endostar release was $24.2 \%$ on day 21 .

\section{Pharmacokinetic characteristics of endostar-loaded nanoparticles}

The standard curve for endostar in plasma was derived from the following equation:

$$
\mathrm{y}=0.01 \mathrm{x}+0.087(\mathrm{r}>0.9994, \mathrm{Y} \text { : optical density }
$$

value of absorbance, $\mathrm{X}$ : the concentration).

The limit of determination was $2 \mu \mathrm{g} / \mathrm{L}$. Mean plasma concentrations in rabbits over time for the free endostar and

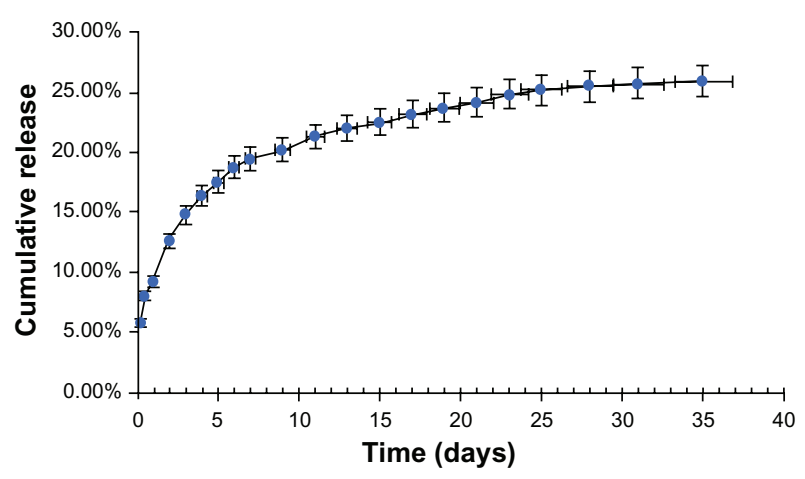

Figure 3 Release of endostar from PEG-PLGA nanoparticles in phosphate-buffered saline $(\mathrm{pH} 7.4,0.01 \%$ sodium azide, $0.02 \%$ Tween 80$)$. The error bars represent mean \pm standard deviations of experiments performed in triplicate.

Abbreviation: PEG-PGLA, poly(ethylene glycol) modified poly(DL-lactideco-glycolide). the endostar-loaded nanoparticles are illustrated in Figure 4. The concentration of endostar in the endostar group was about four times that in the PEG-PLGA nanoparticle group at 10 minutes after intravenous administration. Endostar was removed quickly from the circulation in the endostar group. In contrast, its removal was slow in the endostar-loaded nanoparticle group. The distribution half-life time $\left(\mathrm{t}_{1 / 2 \alpha}\right)$ and terminal elimination half-life $\left(\mathrm{t}_{1 / 2 \beta}\right)$ of endostar were $2.86 \pm 0.53$ hours and $27.95 \pm 8.28$ hours, respectively, in the endostar-containing nanoparticle group, and $0.57 \pm 0.13$ hours and $2.67 \pm 1.30$ hours in the endostar group. The area under the curve was $72628.86 \pm 7522.48 \mathrm{ng} / \mathrm{ml} * \mathrm{~h}$ in the endostar-loaded nanoparticle group and $20574.67 \pm 8081.93 \mathrm{ng} / \mathrm{ml} * \mathrm{~h}$ in the endostar group. The peak concentration $\left(\mathrm{C}_{\max }\right)$ was $3.87 \pm 0.46 \mu \mathrm{g} / \mathrm{mL}$ and $11.81 \pm 4.57 \mu \mathrm{g} / \mathrm{mL}$, respectively. The plasma concentration was $7.4-84.7 \mu \mathrm{g} / \mathrm{L}$ between day 5 and day 10 .

Although data were collected only during the first two days for mice, a similar clearance was shown in the two groups (Figure 5). The $t_{1 / 2 \alpha}$ and $t_{1 / 2 \beta}$ of endostar were $4.83 \pm 1.31$ hours and $24.79 \pm 9.36$ hours, respectively, in the endostar-loaded nanoparticle group, and $0.29 \pm 0.18$ hours and $1.21 \pm 0.96$ hours in the endostar group.

\section{Amount of endostatin in different tissues in vivo}

The relative content of endostatin in the endostar group at 10 minutes was $45.5 \pm 3.5$ in lung, $36.7 \pm 4.2$ in liver, and $48.7 \pm 5.9$ in spleen, and was $133 \pm 19.6,127 \pm 21.7$, and $112 \pm 15.4$, respectively, in the endostar-loaded nanoparticle group. The relative endostatin levels in the endostar-loaded nanoparticle group at three hours was $7.5 \pm 4.1$ in lung, $9.3 \pm 7.2$ in liver, and $13 \pm 11.9$ in spleen, but was not detected in the endostar group (Figure 6). The levels of endostatin

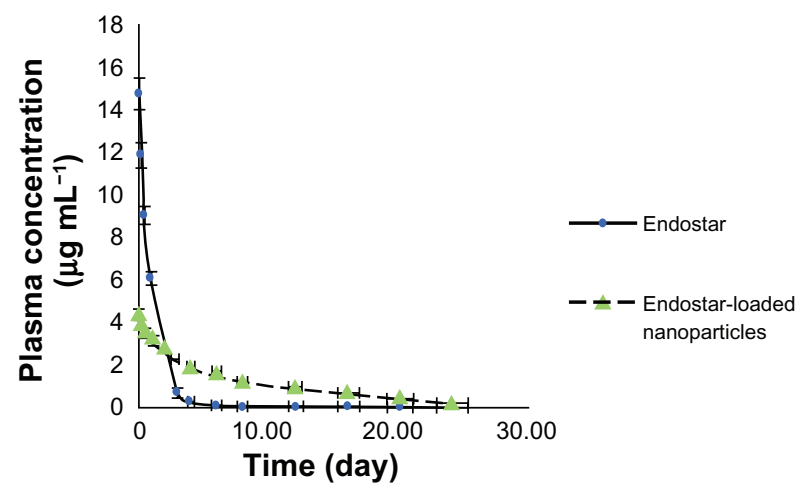

Figure 4 Mean plasma concentration of endostar following a single intravenous administration of endostar or endostar-loaded PEG-PLGA nanoparticles at $90 \mathrm{mg} / \mathrm{m}^{2}$ in rabbits.

Abbreviation: PEG-PGLA, poly(ethylene glycol) modified poly(DL-lactide-coglycolide). 


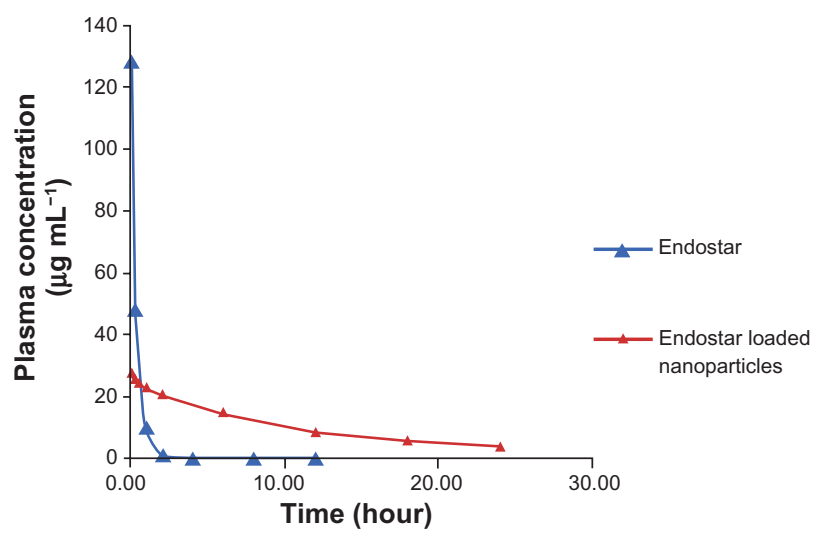

Figure 5 Mean plasma concentration of endostar following a single intravenous administration of endostar or endostar-loaded PEG-PLGA nanoparticles at $90 \mathrm{mg} /$ $\mathrm{m}^{2}$ in mice.

Abbreviation: PEG-PGLA, poly(ethylene glycol) modified poly(DL-lactide-coglycolide).

Ten minutes after dosing

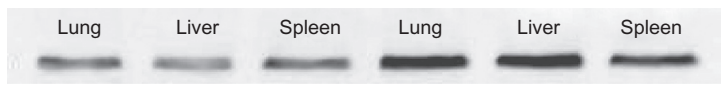

Three hours after dosing

Lung Liver Spleen Lung Liver Spleen

\section{$\beta$-actin}
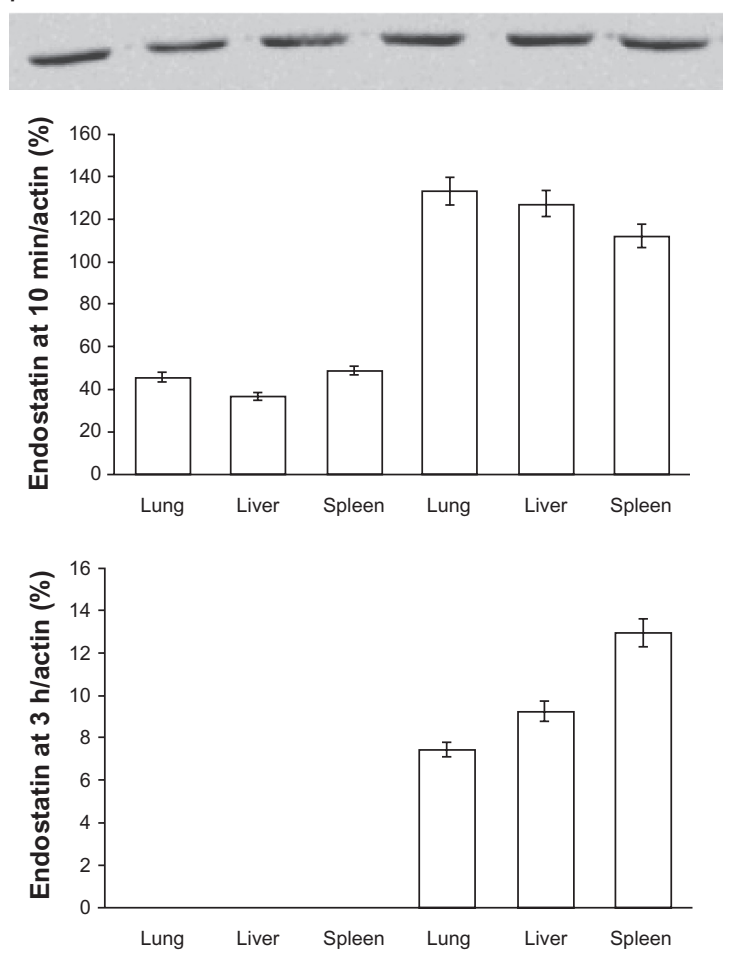

Figure 6 Amount of endostatin in tissues following a single intravenous administration of endostar or endostar-loaded PEG-PLGA nanoparticles. Protein were separated by polyacrylamide gel electrophoresis and probed with antiendostatin antibody (Western blot). The left three bands were from endostar group and the right ones from the endostar-loaded nanoparticle group.

Abbreviation: PEG-PGLA, poly(ethylene glycol) modified poly(DL-lactideco-glycolide). and vascular endothelial growth factor in tumor tissue in the endostar-loaded nanoparticle group were $21.5 \pm 11.9$ and 0 , respectively, and were $10.6 \pm 5.3$ and $4.6 \pm 2.7$ in the endostar group (Figure 7).

\section{Tumor inhibition effect of endostar-loaded nanoparticles in vivo}

The tumor formation rate was $100 \%$ in the study. No deaths and no metastases in organs were found during treatment, and all mice were alive without dyscrasia. There was no significant difference in the body weight of mice between the four groups before and after treatment. All tumors continued to grow after injection, and many started to regress on day 5. As shown by the tumor growth curve of HT-29 cell xenografts, xenograft growth was fast in the blank and control groups, but was significantly depressed in the endostar and endostar nanoparticle-treated groups (Figure 8). Tumor doubling time was prolonged in the treatment group (4.1 days in the endostar group and 5.5 days in endostar-loaded nanoparticle group versus 3.18 days in the blank control group and 3.27 days in the blank nanoparticle group). The inhibition rate was $58.56 \%$ in the endostar group, $77.75 \%$ in the endostar-loaded nanoparticle group, and $8.97 \%$ in the blank nanoparticle group. The tumor
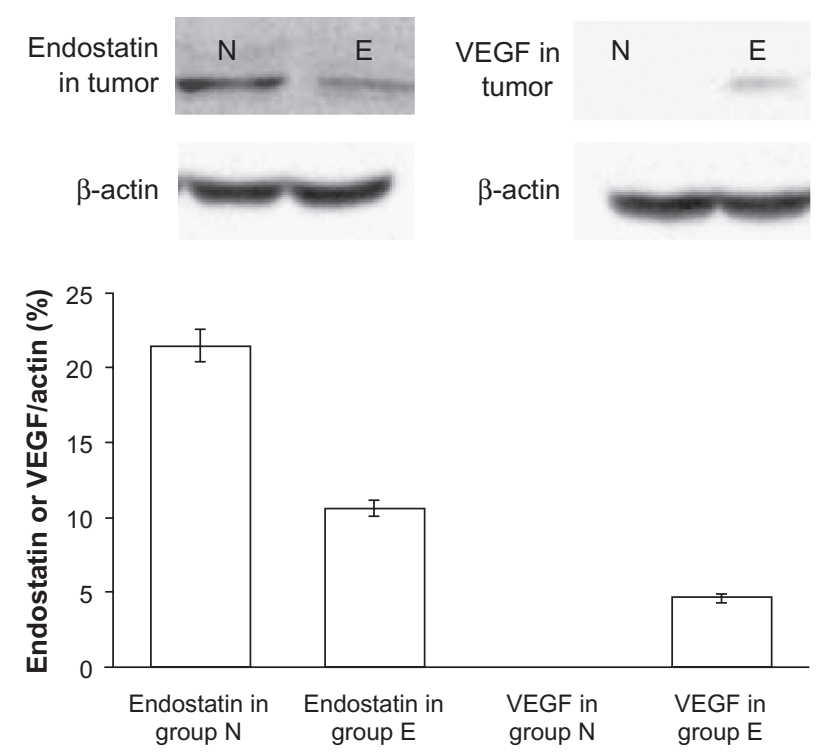

Figure 7 Amounts of endostatin and vascular endothelial growth factor in tumor tissue after treatment with endostar or endostar-loaded PEG-PLGA nanoparticles. Proteins were separated by polyacrylamide gel electrophoresis and probed with antiendostatin or antivascular endothelial growth factor antibody. $\mathrm{N}$ represents the sample from the endostar-loaded nanoparticles group, and $E$ the sample from the endostar group.

Abbreviations: PEG-PGLA, poly(ethylene glycol) modified poly(DL-lactide-coglycolide); VEGF, vascular endothelial growth factor. 


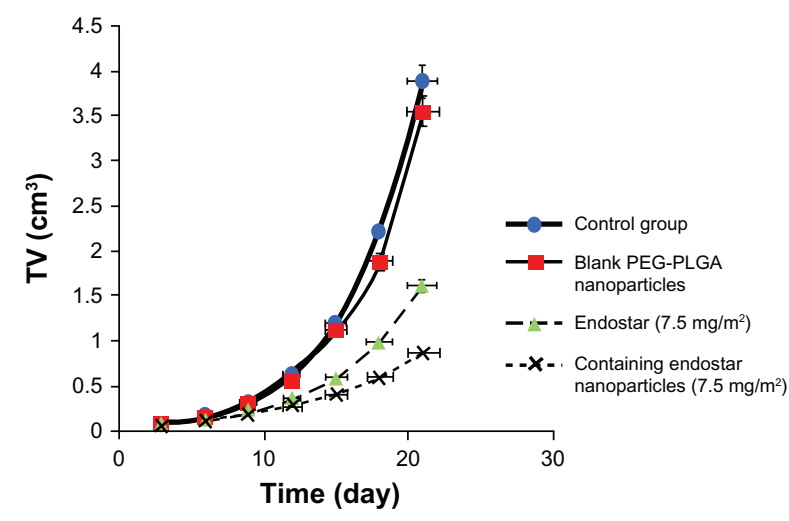

Figure 8 Tumor growth of HT-29 cell xenografts after treatment with endostar or endostar-loaded PEG-PLGA nanoparticles.

Abbreviation: PEG-PGLA, poly(ethylene glycol) modified poly(DL-lactide-coglycolide).

volumes of the endostar, endostar-loaded nanoparticle, blank nanoparticle, and phosphate-buffered saline groups on day 21 were $1.613 \pm 0.448 \mathrm{~cm}^{3}, 0.866 \pm 0.341 \mathrm{~cm}^{3}$, $3.543 \pm 0.640 \mathrm{~cm}^{3}$, and $3.892 \pm 1.076 \mathrm{~cm}^{3}$, respectively (Figure 9). No significant toxicity was observed in any of the four groups.

Microvascular density in the tumors was reduced after treatment. Microscopic observations are shown in Figure 10. The amount of microvessels in the endostar-loaded

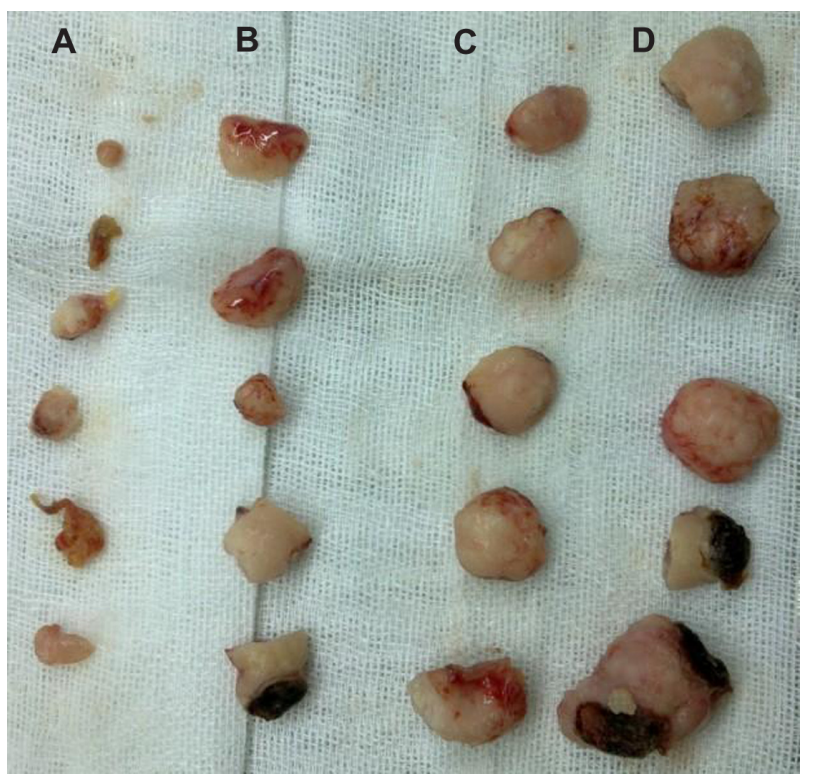

Figure 9 Suppression of HT-29 tumor growth in nude mice. A) Endostar-loaded PEGPLGA nanoparticle group. B) Endostar group. C) Blank PEG-PLGA nanoparticle group. D) Phosphate-buffered saline blank control group. There is no statistically significant difference between $C$ and $D$; however, $A$ and $B$ is significantly different from $D$. Abbreviation: PEG-PGLA, poly(ethylene glycol) modified poly(DL-lactide-coglycolide)

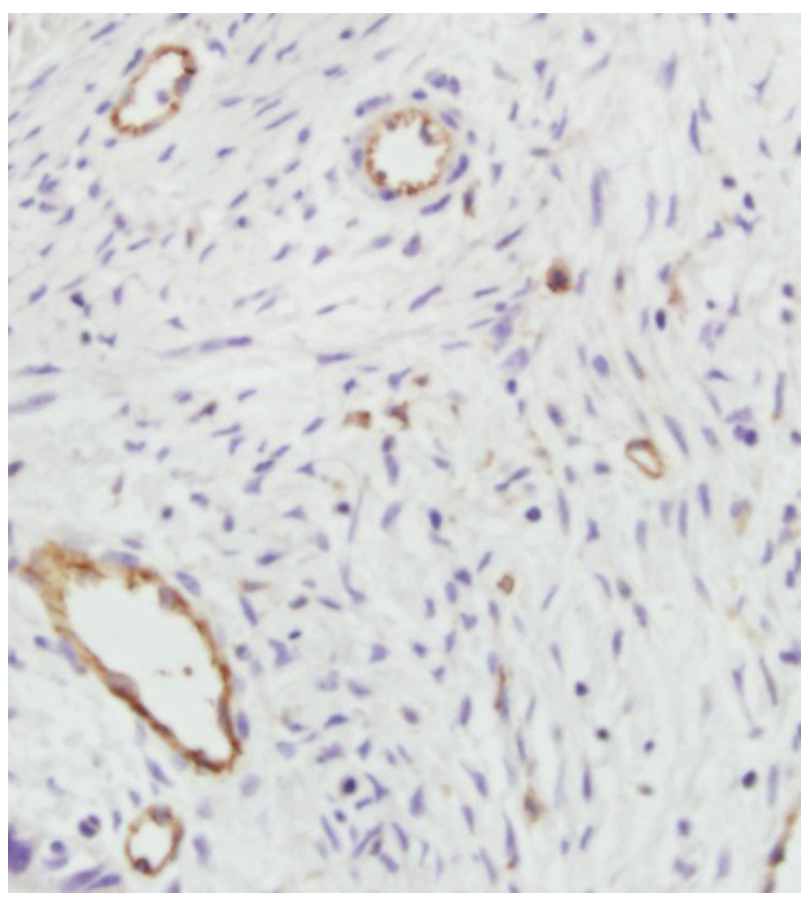

Figure 10 Histologic slice obtained from animal treated with endostar-loaded nanoparticles (CD-3 Istaining, 400×). Vessels appear as dark cycle areas.

nanoparticle group was significantly lower than that in the endostar group ( $6 \pm 2.3$ versus $15 \pm 5.1, P<0.05)$, and was also lower than in the control $(43 \pm 6.7)$ and PEG-PLGA nanoparticle groups $(56 \pm 7.3, P<0.01)$.

\section{Discussion}

Although endostar microsphere and protein drug-loaded PEG-PLGA nanoparticles had been reported, ${ }^{20,21}$ we have prepared a new nanoparticle, ie, the endostar-loaded PEGPLGA nanoparticle, and investigated its characteristics in this study. Endostar-loaded PEG-PLGA nanoparticles are approximately $169.56 \pm 35.03 \mathrm{~nm}$ in diameter. They are smaller than conventional microspheres, can be administered intravenously, and accumulate readily in tumors. It was demonstrated that endothelial cells in tumors were distinct from those in normal tissues, possessing wide fenestrations, ranging from $200 \mathrm{~nm}$ to $1.2 \mathrm{~mm}$. The vascular pore size of the LS174T tumor, a human colon adenocarcinoma, may be as large as $400 \mathrm{~nm}$. This large pore size allows passage of nanoparticles into the extravascular space. ${ }^{22}$ There is increased extravasation and accumulation of drug from the tumor vasculature into the tumor cells, attributed to the enhanced permeability of tumor endothelium and lack of lymphatic drainage in tumor cells. 
Endostar is a $20 \mathrm{kDa}$ peptide and different from protein drugs which are encapsulated within PLGA or PEG-PLGA nanoparticles..$^{21,23}$ It is smaller than a protein molecule and more difficult to encapsulate. Thus, PEG and PLGA, which are hydrophilic-hydrophobic diblock copolymers, were used in this study. They have great potential as vehicles for the delivery of anticancer drugs. ${ }^{24,25}$ PLGA, the hydrophobic moiety, is biodegradable and acts as a drug incorporation site. PEG, the hydrophilic moiety, is a nontoxic, nonimmunogenic, and hydrophilic polymer which can prevent interactions with cells and proteins. ${ }^{26,27}$ Studies have revealed that nanoparticles of $100 \mathrm{~nm}$ in thickness with a PEG layer more than $10 \mathrm{~nm}$ in thickness are not easily engulfed by phagocytes (Figure 11). ${ }^{28,29}$ Because of the hydrophilic moiety, the encapsulation of endostar-loaded nanoparticles was high at $80.17 \% \pm 7.83 \%$.

Moreover, PEG-PLGA nanoparticles hydrolyze in an aqueous environment (hydrolytic degradation or biodegradation). ${ }^{30}$ The biodegradation rates of PLGA copolymers are dependent on the molar ratio of the lactic and glycolic acids in the polymer chain. Thus, PEG-PLGA nanoparticles have been used for controlling the release of drugs, altering pharmacokinetics, enhancing anticancer effect, and decreasing toxicity, especially for water-insoluble drugs. ${ }^{31-33}$ Similar to most proteins, endostar is administered by multiple injections at a high dose in order to maintain adequate therapeutic levels. ${ }^{34,35}$ Clinically, endostar is administered intravenously at a dose of $7.5 \mathrm{mg} / \mathrm{m}^{2}$ per day during the first two weeks of a treatment cycle. However, the plasma concentration of endostar would still fluctuate because of its short biologic half-life and rapid metabolism. In addition, the need for multiple injections causes poor patient compliance, which

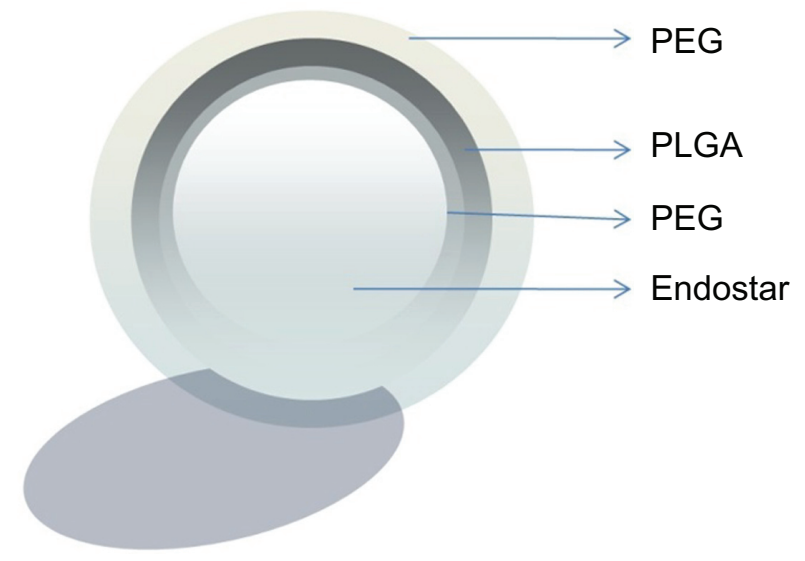

Figure I I Endostar-loaded nanoparticles with a PEG layer. Abbreviation: PEG, poly(ethylene glycol); PGLA, poly(DL-lactide-co-glycolide). limits its clinical use. However, PEG-PLGA nanoparticles change the pharmacokinetic characteristics of endostar, with the $t_{1 / 2 \alpha}$ and $t_{1 / 2 \beta}$ of endostar-containing nanoparticles being longer than for endostar $(P<0.05)$ and the area under the curve and maximum concentration being larger than for endostar $(P<0.05)$.

As shown by Western blot assay, the amounts of endostatin detected in the liver, spleen, and lung in the Endostarcontaining PEG-PLGA nanoparticle group were larger than that in the endostar group after 10 minutes. There was no statistically significant difference between amounts detected in liver and spleen, although the amount of endostatin in lung was higher than in liver or spleen. Endostatin was detected only in the endostar-loaded PEG-PLGA group at three hours after intravenous injection. Even when the mice were sacrificed, the amount of endostatin in tumors for the endostar-loaded nanoparticle group was larger than for the endostar group.

The anticancer effect of endostar was improved when incorporated into PEG-PLGA nanoparticles. As shown in the colon cancer model, tumor doubling time was prolonged in the endostar-loaded nanoparticle group. The inhibition rate for endostar-loaded nanoparticles was higher than for endostar alone $(P<0.05)$. The tumor volumes of the endostar and endostar-loaded nanoparticle-treated groups were significantly smaller than those of the control and blank PEG-PLGA nanoparticle control groups $(P<0.01)$. There was no significant difference in tumor inhibition between the two control groups $(P>0.05)$. Because endostar can inhibit neovascularization or induce apoptosis of vascular endothelium to kill tumor cells, ${ }^{36}$ microvascular density was reduced in tumors after treatment with endostar-loaded nanoparticles. The formation of new capillaries in the existing vasculature is a process fundamental to the development of a solid tumor, and it was hard to elicit angiogenesis in the tumors treated with endostar, and they did not grow fast. The antiangiogenic effect of endostatin is related to vascular endothelial growth factor. ${ }^{8}$ The amount of vascular endothelial growth factor in tumors for the endostar-loaded nanoparticle group was found to be less than for the endostar group when the mice were sacrificed $(P<0.05)$.

PEG-PLGA nanoparticles could maintain adequate concentrations of endostar in plasma and tumor, thereby improving its antitumor effect. PEG-PLGA nanoparticles have a great potential to be protein drug carriers. Although more of their characteristics need to be investigated, and 
some disadvantages need to be overcome, including passive targeting, low drug-loading capacity, and sensitization, PEGPLGA nanoparticles could be modified to be specific for cancer and applied in the clinical setting as a protein carrier system in the future.

\section{Conclusion}

In this study, we prepared endostar-loaded PEG-PLGA nanoparticles in an innovative way and found that they were useful for sustained release of endostar. Although many other characteristics need to be investigated, endostar-loaded PEGPLGA nanoparticles may improve the anticancer activity of endostar by changing the pharmacokinetic behavior of endostar in vivo.

\section{Acknowledgment}

This work was supported by the National High Technology Research and Development Program 863 Fund of China (No.2007AA021802).

\section{Disclosure}

The authors report no conflicts of interest in this work.

\section{References}

1. Michor F, Iwasa Y, Lengauer C, Nowak MA. Dynamics of colorectal cancer. Semin Cancer Biol. 2005;15(6):484-493.

2. Nishiyama N, Kataoka K. Current state, achievements, and future prospects of polymeric micelles as nanocarriers for drug and gene delivery. Pharmacol Ther. 2006;112(3):630-648.

3. Torchilin VP. Targeted pharmaceutical nanocarriers for cancer therapy and imaging. AAPS J. 2007;9(2):E128-E147.

4. Jun YJ, Kim JI, Jun MJ, Sohn YS. Selective tumor targeting by enhanced permeability and retention effect. Synthesis and antitumor activity of polyphosphazene-platinum (II) conjugates. J Inorg Biochem. 2005;99(8):1593-1601.

5. Greish K. Enhanced permeability and retention of macromolecular drugs in solid tumors: A royal gate for targeted anticancer nanomedicines. J Drug Target. 2007;15(7-8):457-464.

6. Iyer AK, Khaled G, Fang J, Maeda H. Exploiting the enhanced permeability and retention effect for tumor targeting. Drug Discov Today. 2006;11(17-18):812-818.

7. Wang C, Pham PT. Polymers for viral gene delivery. Expert Opin Drug Deliv. 2008;5(4):385-401.

8. Ling Y, Yang Y, Lu N, et al. Endostar, a novel recombinant human endostatin, exerts antiangiogenic effect via blocking VEGF-induced tyrosine phosphorylation of KDR/Flk-1 of endothelial cells. Biochem Biophys Res Commun. 2007;361(1):79-84.

9. Zhuo W, Luo C, Wang X, Song X, Fu Y, Luo Y. Endostatin inhibits tumour lymphangiogenesis and lymphatic metastasis via cell surface nucleolin on lymphangiogenic endothelial cells. J Pathol. 2010;222(3):249-260.

10. Song HF, Liu XW, Zhang HN, et al. Pharmacokinetics of His-tag recombinant human endostatin in Rhesus monkeys. Acta Pharmacol Sin. 2005;26(1):124-128.

11. Jiang LP, Zou C, Yuan X, et al. N-terminal modification increases the stability of the recombinant human endostatin in vitro. Biotechnol Appl Biochem. 2009;54(2):113-120.
12. Cohen S, Yoshioka T, Lucarelli M, Hwang LH, Langer R. Controlled delivery systems for proteins based on poly(lactic/glycolic acid) microspheres. Pharm Res. 1991;8(6):713-720.

13. Roy A, Singh MS, Upadhyay PK, Bhaskar S. Combined chemoimmunotherapy as a prospective strategy to combat cancer: A nanoparticle based approach. Mol Pharm. 2010 Sep 16. [Epub ahead of print].

14. Mukherjee B, Santra K, Pattnaik G, Ghosh S. Preparation, characterization and in-vitro evaluation of sustained release protein-loaded nanoparticles based on biodegradable polymers. Int J Nanomedicine. 2008;3(4):487-496.

15. Gref R, Quellec P, Sanchez A, et al. Development and characterization of CyA-loaded poly(lactic acid)-poly(ethylene glycol)PEG micro- and nanoparticles. Comparison with conventional PLA particulate carriers. Eur J Pharm Biopharm. 2001;51(2):111-118.

16. Thomas JP, Arzoomanian RZ, Alberti D, et al. Phase I pharmacokinetic and pharmacodynamic study of recombinant human endostatin in patients with advanced solid tumors. $J$ Clin Oncol. 2003;21(2):223-231.

17. Tentler JJ, Bradshaw-Pierce EL, Serkova NJ, et al. Assessment of the in vivo antitumor effects of ENMD-2076, a novel multitargeted kinase inhibitor, against primary and cell line-derived human colorectal cancer xenograft models. Clin Cancer Res. 2010;16(11): 2989-2998.

18. Jie JZ, Wang JW, Qu JG, Hung T. Suppression of human colon tumor growth by adenoviral vector-mediated NK4 expression in an athymic mouse model. World J Gastroenterol. 2007;13(13):1938-1946.

19. Gao J, Knutsen A, Arbman G, et al. Clinical and biological significance of angiogenesis and lymphangiogenesis in colorectal cancer. Dig Liver Dis. 2009;41(2):116-122.

20. Wu J, Wu L, Xu X, et al. Microspheres made by w/o/o emulsion method with reduced initial burst for long-term delivery of endostar, a novel recombinant human endostatin. J Pharm Sci. 2009;98(6):2051-2058.

21. Li Y, Pei Y, Zhang X, et al. PEGylated PLGA nanoparticles as protein carriers: Synthesis, preparation and biodistribution in rats. $J$ Control Release. 2001;71(2):203-211.

22. Hobbs SK, Monsky WL, Yuan F, et al. Regulation of transport pathways in tumor vessels: Role of tumor type and microenvironment. Proc Natl Acad Sci U S A. 1998;95(8):4607-4612.

23. Son S, Lee WR, Joung YK, et al. Optimized stability retention of a monoclonal antibody in the PLGA nanoparticles. Int J Pharm. 2009;368(1-2):178-185.

24. Mandal D, Chatterjee U. Synthesis and spectroscopy of CdS nanoparticles in amphiphilic diblock copolymer micelles. J Chem Phys. 2007;126(13):134507.

25. Arimura H, Ohya Y, Ouchi T. Formation of core-shell type biodegradable polymeric micelles from amphiphilic poly(aspartic acid)-block-polylactide diblock copolymer. Biomacromolecules. 2005;6(2):720-725.

26. Wang M, Thanou M. Targeting nanoparticles to cancer. Pharmacol Res. 2010;62(2):90-99.

27. Li SD, Huang L. Nanoparticles evading the reticuloendothelial system: Role of the supported bilayer. Biochim Biophys Acta. 2009;1788(10):2259-2266.

28. Jeong YI, Kang MK, Sun HS, et al. All-trans-retinoic acid release from core-shell type nanoparticles of poly(epsilon-caprolactone)/ poly(ethylene glycol) diblock copolymer. Int J Pharm. 2004; 273(1-2): 95-107.

29. Zhang X, Pan SR, Hu HM, et al. Poly(ethylene glycol)-blockpolyethylenimine copolymers as carriers for gene delivery: Effects of PEG molecular weight and PEGylation degree. J Biomed Mater Res A. 2008;84(3):795-804.

30. Muthu M. Nanoparticles based on PLGA and its co-polymer: An overview. Asian J Pharm. 2009;3(4):266-273.

31. Peer D, Karp JM, Hong S, et al. Nanocarriers as an emerging platform for cancer therapy. Nat Nanotechnol. 2007;2(12):751-760. 
32. Wang MD, Shin DM, Simons JW, Nie S. Nanotechnology for targeted cancer therapy. Expert Rev Anticancer Ther. 2007;7(6): 833-837.

33. Petros RA, de Simone JM. Strategies in the design of nanoparticles for therapeutic applications. Nat Rev Drug Discov. 2010;9(8):615-627.

34. Kisker O, Becker CM, Prox D, et al. Continuous administration of endostatin by intraperitoneally implanted osmotic pump improves the efficacy and potency of therapy in a mouse xenograft tumor model. Cancer Res. 2001;61(20):7669-7674.
35. Xu F, Ma Q, Sha H. Optimizing drug delivery for enhancing therapeutic efficacy of recombinant human endostatin in cancer treatment. Crit Rev Ther Drug Carrier Syst. 2007;24(5):445-492.

36. Ling Y, Lu N, Gao Y, et al. Endostar induces apoptotic effects in HUVECs through activation of caspase-3 and decrease of Bcl-2. Anticancer Res. 2009;29(1):411-417.

\section{Publish your work in this journal}

The International Journal of Nanomedicine is an international, peerreviewed journal focusing on the application of nanotechnology in diagnostics, therapeutics, and drug delivery systems throughout the biomedical field. This journal is indexed on PubMed Central, MedLine, CAS, SciSearch $\AA$, Current Contents ${ }^{\circledR} /$ Clinical Medicine,
Journal Citation Reports/Science Edition, EMBase, Scopus and the Elsevier Bibliographic databases. The manuscript management system is completely online and includes a very quick and fair peer-review system, which is all easy to use. Visit http://www.dovepress.com/ testimonials.php to read real quotes from published authors. 